\title{
Lipoxins as biomarkers of lupus and other inflammatory conditions
}

Undurti N Das ${ }^{1,2,3,4}$

\begin{abstract}
Inflammatory events persist in systemic lupus erythematosus (lupus) despite the use of anti-inflammatory (both steroidal and non-steroidal) and immunosuppressive drugs leading to delay in the healing/repair process and so tissue/organ damage continues. The continuation of inflammation in lupus could be attributed to failure of the resolution process due to deficiency of potent endogenous pro-resolution-inducing molecules such as lipoxin $\mathrm{A}_{4}$ $\left(\mathrm{LXA}_{4}\right)$. It is likely that progression and flares of lupus and lupus nephritis are due to decreased formation and release of $L X A_{4}$. Hence, administration of $L X A_{4}$ and its analogues could be of benefit in lupus. Furthermore, plasma and urinary measurement of lipoxins may be used to predict prognosis and response to therapy. It is likely that lipoxins and other bioactive anti-inflammatory lipids such as resolvins, protectins, maresins and nitrolipids play a significant role in other auto-immune diseases such as rheumatoid arthritis, type 1 diabetes mellitus and multiple sclerosis and hence, could be of significant benefit in these diseases.
\end{abstract}

Keywords: Lupus rheumatoid arthritis, diabetes mellitus, multiple sclerosis, lipoxins, resolvins, protectins, maresins, autoimmunity, cytokines, tumor necrosis factor, free radicals, prostaglandins

\section{Introduction}

Systemic lupus erythematosus (SLE), a disease of unknown aetiology that is more common in women than in men, is characterized by non-destructive arthritis/arthralgias, a cutaneous rash, vasculitis, involvement of the central nervous system (CNS) and renal and cardiopulmonary manifestations. Although genetic, environmental and sex hormonal factors have been implicated in the pathogenesis of SLE (also called as "lupus"), it is known that several cytokines, nitric oxide (NO), free radicals, a deranged immune system, a deficient anti-oxidant defenses, and Toll-like receptors have a significant role both in the initiation and perpetuation of the inflammatory process observed.

The fundamental process in lupus appears to be rendering DNA and RNA antigenic that leads to the production of anti-DNA and anti-RNA antibodies and the formation of immune complexes. These antibodies and immune complexes, in turn, trigger both a local and systemic inflammatory response that ultimately leads to

\footnotetext{
Correspondence: undurti@hotmail.com

'UND Life Sciences, 13800 Fairhill Road, \#321, Shaker Heights, OH 44120, USA
}

Full list of author information is available at the end of the article target organ/tissue damage seen in lupus. The susceptibility to develop lupus in a given individual seems to have, at least, partly a genetic basis though this is still not very clear. Once the inflammatory process is triggered, this leads to the production of a variety of pro-inflammatory cytokines such as interleukin-1 (IL-1), IL-6, tumor necrosis factor- $\alpha$ (TNF- $\alpha$ ), interferons (IFNs), macrophage migration inhibitory factor (MIF), HMGB1 (high mobility group B1) and possibly, a reduction in the elaboration of anti-inflammatory cytokines such as IL-10, IL-4, and transforming growth factor- $\beta$ (TGF- $\beta$ ). This imbalance between the pro- and antiinflammatory cytokines coupled with increased secretion of free radicals such as superoxide anion $\left(\mathrm{O}_{2}{ }^{-}\right.$.), hydrogen peroxide $\left(\mathrm{H}_{2} \mathrm{O}_{2}\right)$, singlet oxygen, inducible nitric oxide (iNO), and other reactive oxygen species (ROS) by activated monocytes, macrophages, polymorphonuclear leukocytes (PMNL), T cells, Kupffer cells, glial cells in the brain, and other organ specific reticuloendothelial cells would ultimately cause target tissue/organ damage seen in lupus.

I propose that continued inflammatory events seen in lupus could be due to failure of the resolution of inflammation. Thus, the balance between inflammation and 
resolution is disturbed more in favor of pro-inflammatory events and/or failure of resolution inducing molecules to be produced at the most appropriate time leading to non-resolution of inflammation. In other words, even after the inciting agent responsible for the initiation of inflammation is removed; inappropriate inflammation continues simply because resolution failed to occur. This leads to delay in the healing/repair process and so tissue/organ damage continues. This may explain as to why even when these patients are continuing to take anti-inflammatory and immunosuppressive medicines, target organ damage continues. In view of this, it is imperative that institution of pro-resolutioninducing agents needs to be employed to obtain full remission and restore normal physiological function of the target tissues/organs in these diseases. Such endogenous pro-resolution-inducing molecules include: lipoxins, resolvins, protectins, maresins, nitric oxide, nitrolipids, 15 deoxy $\Delta^{12-14} \mathrm{PGJ}_{2}, \mathrm{PGD}_{2}$, anti-inflammatory cytokines such as IL-4, IL-10, and some polyunsaturated fatty acids (PUFAs).

Based on this hypothesis, it is suggested that progression and flares of lupus are due to increased production of pro-inflammatory molecules IL-6, TNF- $\alpha$, MIF (macrophage migration inhibitory factor), HMGB1 (high mobility group box 1), free radicals and lipid mediators such as prostaglandins (PGs), leukotrienes (LTs) and/or decreased formation and release of anti-inflammatory molecules: IL-4, IL-10, TGF- $\beta$, and lipoxins, resolvins, protectins, maresins and nitrolipids.

\section{Metabolism of essential fatty acids with specific reference to inflammation}

Cis-Linoleic acid (LA, 18:2 $\omega-6)$ and $\alpha$-linolenic acid (ALA, 18:3 $\omega-3$ ) are essential nutrients since they cannot be synthesized by the human body and hence are called as "essential fatty acids" (EFAs). LA is converted to gamma-linolenic acid (GLA, 18:3, $\omega-6$ ) by the action of the enzyme $\Delta^{6}$ desaturase, and GLA is elongated to form di-homo-GLA (DGLA, 20:3, $\omega-6$ ), the precursor of the 1 series of prostaglandins. $\Delta^{6}$ desaturase is the ratelimiting step in the metabolism of EFAs. DGLA can also be converted to arachidonic acid (AA, 20:4, $\omega-6)$ ) by the action of the enzyme $\Delta^{5}$ desaturase. AA forms the precursor of 2 series of prostaglandins, thromboxanes and the 4 series leukotrienes (LTs). ALA is converted to eicosapentaenoic acid (EPA, 20:5, $\omega-3$ ) by $\Delta^{6}$ and $\Delta^{5}$ desaturases. EPA forms the precursor of the 3 series of prostaglandins and the 5 series of LTs. EPA can be elongated to form docosahexaenoic acid (DHA, 22:6, $\omega$ 3). AA, EPA and DHA also form precursors to a group of novel compounds: lipoxins, resolvins, protectins and maresins [1-9] that have anti-inflammatory action. Eicosanoids bind to $\mathrm{G}$ protein-coupled receptors on many cell types and mediate virtually every step of inflammation, are found in inflammatory exudates, and their synthesis is increased at sites of inflammation. Non-steroidal anti-inflammatory drugs (NSAIDs) such as aspirin inhibit cyclo-oxygenase (COX) activity and thus, are believed to bring about their anti-inflammatory action.

\section{Lipoxins, resolvins, protectins and maresins}

There are two cyclo-oxygenase enzymes, the constitutively expressed COX-1 and the inducible enzyme COX2. Different types of PGs are formed by the action of COX enzymes depending on the substrate fatty acid from which they are derived. Different types of PGs have different actions and sometimes diametrically opposite actions. For example, $\mathrm{PGE}_{2}, \mathrm{PGF}_{2 \alpha}$, thromboxane $\mathrm{A}_{2}\left(\mathrm{TXA}_{2}\right)$, and leukotrienes (LTs) have pro-inflammatory actions whereas $\mathrm{PGE}_{1}$ and prostacyclin $\left(\mathrm{PGI}_{2}\right)$ show anti-inflammatory actions. Furthermore, the distributions of COX-1 and COX-2 enzymes have restricted tissue distribution. Platelets contain thromboxane synthetase, and hence $\mathrm{TXA}_{2}$, a potent platelet-aggregator and vasoconstrictor, is formed in these cells, whereas vascular endothelial cells possess $\mathrm{PGI}_{2}$ synthetase but lack thromboxane synthetase and thus, they form, mainly, $\mathrm{PGI}_{2}$, a potent platelet anti-aggregator and vasodilator. The balance between $\mathrm{TXA}_{2}$ and $\mathrm{PGI}_{2}$ is important in thrombus formation in coronary and cerebral blood vessels. $\mathrm{PGD}_{2}, \mathrm{PGE}_{2}$ and $\mathrm{PGF}_{2 \alpha}$, major metabolites of the COX pathway have pro-inflammatory actions.

There are 3 types of lipoxygenases and are present in only a few types of cells. 5-lipoxygenase (5-LO), present in neutrophils, produces 5-HETE, which is chemotactic for neutrophils, and is converted into leukotrienes (LTs). $\mathrm{LTB}_{4}$, a potent chemotactic and activator of neutrophils, induces aggregation and adhesion of leukocytes to vascular endothelium, generation of ROS, and release of lysosomal enzymes. The cysteinyl-containing leukotrienes $\mathrm{C}_{4}, \mathrm{D}_{4}$, and $\mathrm{E}_{4}\left(\mathrm{LTC}_{4}, \mathrm{LTD}_{4}\right.$, and $\left.\mathrm{LTE}_{4}\right)$ induce vasoconstriction, bronchospasm, and vascular permeability in venules. LTs are more potent than histamine in increasing vascular permeability and causing bronchospasm. LTs mediate their actions by binding to cysteiny leukotreine 1 (CysLT1) and CysLT2 receptors.

Lipoxins (LXs) are generated from AA, EPA and DHA by transcellular biosynthetic mechanisms involving two cell populations. Neutrophils produce intermediates in LX synthesis, and these are converted to LXs by platelets interacting with leukocytes. $\mathrm{LXA}_{4}$ and $\mathrm{LXB}_{4}$ are generated by the action of platelet 12-lipoxygenase on neutrophil-derived $\mathrm{LTA}_{4}$. LXs inhibit leukocyte recruitment, neutrophil chemotaxis and adhesion to endothelium [7]. LXs have a negative regulation on LT synthesis and action and help in the resolution of inflammation. 
An inverse relationship generally exists between LXs and LTs and the balance between these two molecules appears to be crucial in the determination of degree of inflammation and its final resolution $[4,6]$.

\section{Aspirin-triggered 15 epimer LXs (ATLs), resolvins and protectins}

The formation of aspirin-triggered 15 epimer LXs (ATLs) are potent counter regulators of polymorphonuclear neutrophils (PMNs)-mediated injury and acute inflammation. Acetylated COX-2 enzyme of endothelial cells generates 15R-hydroxyeicosatetraenoic acid (15RHETE) from AA that is converted by activated PMNs to the 15-epimeric LXs that have potent anti-inflammatory properties [2-9]. This cross-talk between endothelial cells and PMNs leading to the formation of 15R-HETE and its subsequent conversion to 15-epimeric LXs by aspirin-acetylated COX-2 is a protective mechanism to prevent local inflammation on the vessel wall by regulating the motility of PMNs, eosinophils, and monocytes [9]. Endothelial cells also oxidize AA, EPA and DHA via P450 enzyme system to form various hydroxyeicosatetraenoic acids and epoxyeicosatrienoic acids such as 11,12-epoxy-eicosatetraenoic acid(s) that have many biological actions that include blocking endothelial cell activation, while non-enzymatic oxidation products of EPA inhibit phagocyte-endothelium interaction and suppress the expression of adhesion molecules [10-15].

Akin to the formation of 15R-HETE and 15-epimeric LXs from AA, similar compounds are also formed from EPA and DHA. In the presence of aspirin, activated COX-2 of human endothelial cells converts EPA to 18R-HEPE, 18-HEPE, and 15R-HEPE. Activated human PMNs, in turn, converted 18R-HEPE to 5,12,18R-triHEPE and 15R-HEPE to 15-epi-LXA Ly $_{5}$ their 5-lipoxygenase. Both 18R-HEPE and 5,12,18R-triHEPE inhibited $\mathrm{LTB}_{4}$-stimulated PMN transendothelial migration. 5,12,18R-triHEPE effectively competed with $\mathrm{LTB}_{4}$ for its receptors and inhibited PMN infiltration suggesting that it suppresses LT-mediated responses at the sites of inflammation $[4,6,16,17]$.

The conversion of EPA by human endothelial cells with upregulated COX-2 treated with ASA of EPA to 15-epi-LX, also termed aspirin-triggered LX [ATL] and to 18R-hydroxyeicosapentaenoic acid (HEPE) and 15RHEPE is interesting. These compounds in turn, are used by polymorphonuclear leukocytes to generate separate classes of novel trihydroxy-containing mediators, including 5-series 15R-LX(5) and 5,12,18R-triHEPE, which are potent inhibitors of human polymorphonuclear leukocyte transendothelial migration and infiltration in vivo (ATL analogue > 5,12,18R-triHEPE > 18R-HEPE). Acetaminophen and indomethacin also permitted 18R-HEPE and 15R-HEPE generation with recombinant COX-2.
The formation of these bioactive lipid mediators via COX-2-nonsteroidal antiinflammatory drug-dependent oxygenations and cell-cell interactions may have significant therapeutic benefits in inflammation $[16,17]$.

Murine brain cells expressing COX-2 and treated with aspirin transformed enzymatically DHA to $17 \mathrm{R}$ series of hydroxy DHAs (HDHAs) that, in turn, is converted enzymatically by PMNs to di- and tri-hydroxy containing docosanoids [16-19]. The conversion of DHA by leukocytes, brain, and glial cells to 17S-hydroxy-containing docosanoids denoted as docosatrienes (the main bioactive member of the series was 10,17S-docosatriene) and 17S series resolvins serve as regulators of both leukocytes reducing infiltration in vivo and glial cells blocking their cytokine production. These results indicate that DHA is the precursor to potent protective mediators generated via enzymatic oxygenations to novel docosatrienes and $17 \mathrm{~S}$ series resolvins that have significant anti-inflammatory action and participate in the resolution of inflammatory events.

Similar small molecular weight compounds are also generated from AA, EPA, and DHA: 15R-hydroxy containing compounds from AA, 18R series from EPA, and 17R-hydroxy series from DHA. All these compounds have potent anti-inflammatory actions, resolve inflammation and hence are called as "resolvins". Resolvins inhibited cytokine generation, leukocyte recruitment, leukocyte diapedesis, and exudate formation. The formation of resolvins from AA, EPA, and DHA from acetylated COX-2 are generated via transcellular biosynthesis (e.g. due to cell-cell communication between endothelial cells and PMNs), and their main purpose appears to be to suppress inflammation. Resolvins inhibited brain ischemia-reperfusion injury $[18,19]$. It is possible that lipoxins, resolvins and protectins (docosanoids are also called as protectins since they have neuroprotective actions) behave as endogenous anti-inflammatory and cytoprotective molecules. The general cytoprotective properties that have been attributed to AA, EPA, and DHA can be related to their conversion to lipoxins, resolvins and docosanoids (protectins). Hence, any defect in the synthesis of lipoxins, resolvins and protectins or their inappropriate degradation could lead to perpetuation of inflammation as seen in lupus and other rheumatological conditions.

\section{Anti-inflammatory molecule lipoxin $A_{4}$ is detectable in urine}

$\mathrm{LX}_{4}$, generated by lipoxygenase (LO) transformation of AA possess potent anti-inflammatory activity in vivo, and temporal biosynthesis of LX, concurrent with spontaneous resolution, has been observed during exudate formation [8]. Recently, a new extraction technique, more selective for LX, which abolishes background 
contamination and minimizes the unspecific reading, was developed. This new method showed that urine from healthy subjects contains $\mathrm{LXA}_{4}$ [20]. Subsequently, it was shown [21] that strenuous exercise significantly increased urinary excretion in healthy volunteers $(0.061$ \pm 0.023 vs. $0.113 \pm 0.057 \mathrm{ng} / \mathrm{mg}$ creatinine; $\mathrm{P}=0.028$ ). These findings confirm that alterations in the urinary excretion of $\mathrm{LXA}_{4}$ can used as a reflection of changes in its $\left(\mathrm{LXA}_{4}\right)$ formation, especially in the kidney, to monitor changes in the systemic and renal inflammatory lesions.

In a further extension of this work, it was reported that urinary levels of $\mathrm{LXA}_{4}$ was decreased while that of cysLTs (cysteinyl leukotrienes) increased in volunteers aged from 26 to over 100 years leading to a profound unbalance of the LXA(4)/cysLTs ratio that may be considered an index of the endogenous anti-inflammatory potential [22]. These results suggest that endogenous anti-inflammatory mechanisms become less efficient with age that could result in increased susceptibility to inflammatory disorders with advancing age.

\section{Urinary $\mathrm{LXA}_{4}$ is decreased and LTs increased in HSP nephritis}

In a study in which temporal changes of blood and urinary $\mathrm{LXA}_{4}, \mathrm{LTB}_{4}$ (leukotriene $\mathrm{B}_{4}$ ) and urinary $\mathrm{LTE}_{4}$ was determined in 49 children with Henoch-Schonlein purpura (HSP) in which renal inflammation is known to occur, it was reported that inverse temporal changes between gradually increased blood and urinary $\mathrm{LXA}_{4}$ and gradually decreased blood and urinary $\mathrm{LTB}_{4}$ and urinary $\mathrm{LTE}_{4}$. Furthermore, both 15-S-hydroxyeicosatetraenoic acid and $\mathrm{LXA}_{4}$ inhibited the $\mathrm{LTB}_{4}$-induced chemotaxis of leukocytes and release of $\mathrm{LTB}_{4}$ from leukocytes obtained from the patients in the active phase of HSP. In 22 children with HSP nephritis, concordant with the gradually increased severity of mesangial proliferation and proteinuria, the glomerular expressions of 15-lipoxygenase and the levels of urinary $\mathrm{LXA}_{4}$ gradually decreased and the glomerular expressions of $\mathrm{LTC}_{4}$ synthase and the urinary $\mathrm{LTE}_{4}$ and $\mathrm{LTB}_{4}$ gradually increased. The levels of blood and urinary $\mathrm{LXA}_{4}$ in patients with HSP nephritis were lower than those in patients with purpura alone in early resolution of HSP. The levels of blood and urinary $\mathrm{LTB}_{4}$ and urinary $\mathrm{LTE}_{4}$ in the patients with HSP nephritis were higher than those in patients with purpura alone in early resolution of HSP. A positive correlation between blood $\mathrm{LTB}_{4}$ and serum C-reactive protein in 49 children with HSP was also noted [23]. These data suggest that LTs play a proinflammatory and profibrotic role in the pathogenesis of HSP, and insufficiency of $\mathrm{LXA}_{4}$ may be responsible for the patients with HSP whose illness become more serious. These results suggest that absence or decreased levels of $\mathrm{LXA}_{4}$ that acts as a "stop signal" of inflammatory process may be responsible for the nephritis seen in HSP.

\section{Anti-inflammatory cytokines IL-4 and IL-10 enhance LXA synthesis}

In this context, it is noteworthy that anti-inflammatory cytokines IL-4 and IL-10 trigger the conversion of AA, EPA and DHA to lipoxins, resolvins, protectins and maresins suggesting a mechanism by which they are able to suppress inflammation [24]. For example, IL-4 up-regulated \{(it may be noted here that 15-S-HETE (a 15-LO product; LO = lipoxygenase enzyme) and lipoxins (interaction products between 5-LO and either 12-LO or 15-LO) counteract the proinflammatory actions of leukotrienes\} 15-LO gene expression in human leukocytes. Glomerular 12/15-LO mRNA increased significantly over controls 24 and 48 hours after nephrotoxic serum injection then decreased at 72 hours. RNA from nephrotoxic serum injected glomeruli contained higher levels of 12/15-LO mRNA than that from unstimulated peripheral leukocytes, suggesting that $12 / 15$-LO transcription is up-regulated locally in native and/or infiltrating glomerular cells. Glomerular IL-4 mRNA increased markedly 16 hours post-nephrotoxic serum injection, and was then reduced, suggesting a potential role for $\mathrm{T}$ cellderived IL-4 in directing the expression of $12 / 15-\mathrm{LO}$ during glomerulonephritis. This suggested tandem regulated in vivo gene expression for IL-4 and LO, both of which promote counter-inflammatory influences in immune complex-mediated injury.

\section{Balance between $\mathrm{LXA}_{4}$ and LTs determines the degree of systemic and glomerular inflammation}

The 5-lipoxygenated metabolites of AA, the leukotrienes, are major mediators of early glomerular hemodynamic and structural deterioration during experimental glomerulonephritis that are generated largely by infiltrating leukocytes, but can also occur by intrinsic glomerular cells via transcellular metabolism of intermediates. In animal models of glomerulonephritis and other renal pathologic states, leukotrienes have been shown to exert adverse effects in the glomerulus. $\mathrm{LTB}_{4}$ augments neutrophil infiltration, and $\mathrm{LTC}_{4}$ and $\mathrm{LTD}_{4}$ mediate potent vasoconstrictor effects on the glomerular microcirculation. Selective blockade of the 5-lipoxygenase pathway produced a significant amelioration of the deterioration of renal hemodynamic and structural parameters. On the other hand, 15-S-hydroxyeicosatetraenoic acid (15-S-HETE), the immediate product of arachidonate 15lipoxygenase, and the lipoxins, which are produced by sequential 15- and 5- or 5- and 12-lipoxygenation of AA are also generated in the course of glomerular injury that antagonize leukotriene-induced neutrophil chemotaxis 
and lipoxin $\mathrm{A}_{4}$ antagonized the effects of $\mathrm{LTD}_{4}$ and $\mathrm{LTC}_{4}$ on the glomerular microcirculation. Thus, the contrasting effects of 5- and 15-lipoxygenase products represent endogenous pro- and anti-inflammatory influences that ultimately determine and regulate the extent and severity of glomerular inflammation [25-28].

These results are in favor of the proposal that antiinflammatory cytokines IL-4 and IL-10 induce the expression and synthesis of anti-inflammatory lipid mediators lipoxins, resolvins, protectins and maresins in addition to their ability to suppress the production of pro-inflammatory cytokines such as IL-2, IL-6, TNF- $\alpha$, MIF and HMGB1 and LTs.

\section{PUFAs and lipoxins bind to GPCR to suppress inflammation}

It is noteworthy that monocytes and macrophages express an extensive repertoire of $\mathrm{G}$ protein-coupled receptors (GPCRs) that regulate inflammation and immunity. A number of GPCRs (e.g. Edg5, P2ry 2 and 6) have been reported to be expressed by macrophages, and two cell types closely related to macrophages (osteoclasts and dendritic cells), whereas Gpr84 expression was largely restricted to macrophage populations and granulocytes [29]. It is now apparent that many PUFAs, especially AA, EPA and DHA and their metabolites such as eicosanoids, lipoxins, resolvins, protectins and maresins also function directly as agonists at a number of $\mathrm{G}$ protein-coupled receptors (GPCRs). Tissue distribution studies and siRNA knock-down experiments have indicated key roles for these GPCRs in glucose homeostasis, adipogenesis, leukocyte recruitment and inflammation [30]. A recent study showed that the G protein-coupled receptor 120 (GPR120) functions as a $\omega-3$ fatty acid receptor/sensor. Stimulation of GPR120 with $\omega-3$ fatty acids (EPA and DHA) induced broad anti-inflammatory effects in monocytic RAW 264.7 cells and in primary intraperitoneal macrophages. All of these effects were abrogated by GPR120 knockdown. The $\omega-3$ fatty acid treatment not only inhibited inflammation but also enhanced systemic insulin sensitivity in wild-type mice, but was without effect in GPR120 knockout mice. These results suggest that GPR120 is a functional $\omega-3$ fatty acid receptor/ sensor and mediates potent insulin sensitizing and antidiabetic effects in vivo by repressing macrophageinduced tissue inflammation [31]. Thus, it is likely that PUFAs and their anti-inflammatory products such as lipoxins, resolvins, protectins and maresins inhibit the production of various pro-inflammatory molecules including MIF and HMGB1 and thus, suppress inflammation in diseases such as lupus and rheumatoid arthritis.

\section{Hypothesis \\ A deficiency of $L X A_{4}$ and excess of $L T s$ may be responsible for lupus/lupus nephritis}

Based on the evidences presented above and the role of $\mathrm{LXA}_{4}$ and LTs in inflammation, it is quite logical to suggest that a deficiency of $\mathrm{LXA}_{4}$ and/or an excess of LTs initiate and perpetuate systemic and renal inflammation in lupus. Since, it is possible to estimate these compounds in the urine; I propose that progression and flares of lupus and lupus nephritis are due to decreased production of $\mathrm{LXA}_{4}$ and enhanced production of LTs by the renal tissue and/or infiltrating leukocytes and macrophages. These molecules can be detected and estimated in the urine [20-23] as already discussed above. Furthermore, the urinary levels of $\mathrm{LXA}_{4}$ and LTs may also be used to predict prognosis and response to treatment. If the urinary $\mathrm{LXA}_{4}$ levels revert to normal or are slowly increasing with and without decrease in urinary levels of LTs, it can be considered as an indication that the patient is responding to treatment and that both systemic and renal lesions of lupus are ameliorating. The urinary levels of $\mathrm{LXA}_{4}$ and LTs can be compared to their plasma concentrations; and to serum urea, creatinine and urinary protein values and wherever possible to renal biopsy findings to know the progress of renal lupus disease.

Since resolvins, protectins and maresins are also antiinflammatory lipid molecules derived from EPA and DHA and have a role in the resolution of inflammation, it is predicted that they may also have the same significance as that of lipoxins in lupus nephritis. To measure resolvins, protectins and maresins in urine reliable methods are yet to be developed. If they are developed, then it is worthwhile to measure the urinary levels of resolvins, protectins and maresins in addition to lipoxins in the urine of patients with lupus and lupus nephritis to predict prognosis and response to treatment. Decrease in their levels in the urine indicates continued inflammation and renal damage whereas enhancement in their levels indicates resolution of inflammation and amelioration of lupus nephritis.

\section{Therapeutic implications}

Based on this hypothesis, it is proposed that $\mathrm{LXA}_{4}$ and its more stable synthetic analogues and resolvins, protectins, maresins and their agonists are useful in the management of lupus, rheumatoid arthritis, type 1 diabetes mellitus and other autoimmune diseases. Measurement of plasma and urinary levels of $\mathrm{LXA}_{4}$, resolvins, protectins and maresins may be used as prognostic markers in these diseases, while in multiple sclerosis; measurement of cerebrospinal fluid (CSF) levels of $\mathrm{LXA}_{4}$, resolvins, protectins and maresins can be used as 


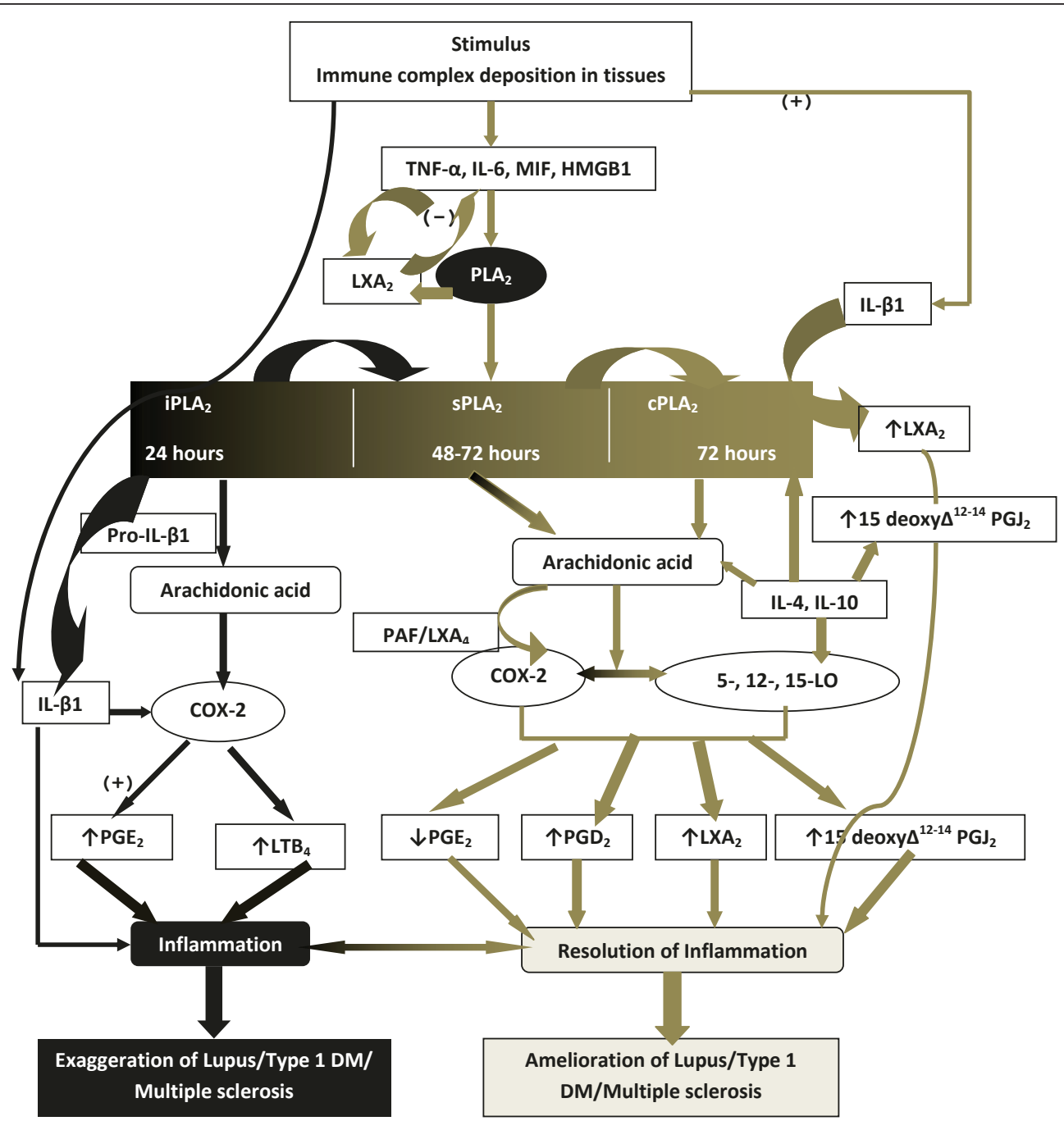

Figure 1 Scheme showing the role of prostaglandins, leukotrienes and lipoxins in lupus and other autoimmune diseases. (-) Indicates inhibition or suppression of action; (+) Indicates activation or enhancement of action. There are three classes of phospholipases that control the release of $A A$ and other PUFAs: calcium-independent PLA $\left(\mathrm{PLA}_{2}\right)$, secretory $P L A_{2}\left(s P L A_{2}\right)$, and cytosolic PLA $\left(C P L A_{2}\right)[40]$. Each class of PLA 2 is further divided into isoenzymes for which there are 10 for mammalian $s P L A_{2}$, at least 3 for $C P L A_{2}$, and 2 for $i P L A_{2}$. During the early phase of inflammation, COX-derived PGs and lipoxygenase-derived LTs initiate exudate formation and inflammatory cell influx [41]. TNF- $\alpha$ causes an immediate influx of neutrophils concomitant with $\mathrm{PGE}_{2}$ and $\mathrm{LTB}_{4}$ production, whereas during the phase of resolution of inflammation an increase in $L X A_{4}$ (lipoxin $A_{4}$ ), $P G D_{2}$ and its product 15deoxy $\triangle^{12-14} P G J_{2}$ formation occurs that induces resolution of inflammation with a simultaneous decrease in $\mathrm{PGE}_{2}$ synthesis that stops neutrophil influx and enhances phagocytosis of debris $[42,43]$. Thus, there appears to be two waves of release of AA and other PUFAs: one at the onset of inflammation that causes the synthesis and release of PGE ${ }_{2}$ and a second at resolution for the synthesis of anti-inflammatory $\mathrm{PGD}_{2}, 15$ deoxy $\triangle^{12-14} P G J_{2}$, and lipoxins that are necessary for the suppression of inflammation. Thus, COX-2 enzyme has both harmful and useful actions by virtue of its ability to give rise to pro-inflammatory and anti-inflammatory PGs and LXs.Increased type VI iPLA 2 protein expression was found to be the principal isoform expressed from the onset of inflammation up to 24 hours, whereas type lla and $V$ sPLA 2 was expressed from the beginning of 48 hours till 72 hours while type IV CPLA $A_{2}$ was not detectable during the early phase of acute inflammation but increased progressively during resolution peaking at 72 hours. This increase in type IV $C P L A_{2}$ was mirrored by a parallel increase in COX-2 expression $[44]^{5}$. The increase in $C P L A_{2}$ and COX-2 occurred in parallel, suggesting a close enzymatic coupling between these two. Thus, there is a clear-cut role for different types of PLA $A_{2}$ in distinct and different phases of inflammation. Selective inhibition of $C P L A_{2}$ resulted in the reduction of pro-inflammatory molecules $P G E_{2}, L T B_{4}, I L-1 \beta$, and platelet-activating factor (PAF). Furthermore, inhibition of types 1 la and $V$ SPLA 2 not only decreased PAF and $L X A_{4}$ (lipoxin $A_{4}$ ) but also resulted in a reduction in CPLA $A_{2}$ and COX-2 activities. These results suggest that sPLA $A_{2}$-derived PAF and LXA 4 induce COX-2 and type IV CPLA $2.1 \mathrm{~L}-1 \beta$ induced CPLA 2 expression. This suggests that one of the functions of IL-1 is not only to induce inflammation but also to induce CPLA 2 expression to initiate resolution of inflammation [45,46].Synthetic glucocorticoid dexamethasone inhibited both CPLA $A_{2}$ and SPLA $A_{2}$ expression, whereas type IV iPLA 2 expression is refractory to its suppressive actions $[44,47,48]$. Activated $\mathrm{PLA} \mathrm{A}_{2}$ contributes to the conversion of inactive pro-IL-1 $\beta$ to active IL-1 $\beta$, which in turn induces $C P L A_{2}$ expression that is necessary for resolution of inflammation.LXs, especially LXA inhibit TNF- $\alpha$-induced production of ILs; promote TNF- $\alpha$ mRNA decay, TNF- $\alpha$ secretion, and leukocyte trafficking and thus attenuates inflammation. 
markers of disease progression, response to therapy and its prognosis.

Previously, I showed that AA, EPA and DHA prevented chemical-induced type $1 \mathrm{DM}$ in experimental animals [32-34]. Recent studies [35-39] both in experimental animals and humans confirmed that PUFAs, especially n-3 fatty acids, do prevent type 1 DM. Since AA, EPA and DHA form precursors to LXs, resolvins, protectins and maresins, the ability of these fatty acids to prevent DM can be ascribed to the formation of the anti-inflammatory lipids [37-39]. Thus, PUFAs and LXs, resolvins, protectins and maresins are expected to be beneficial in lupus, RA, multiple sclerosis and other autoimmune diseases (see Figure 1).

\section{Acknowledgements}

Dr. U N Das is in receipt of Ramalingaswami fellowship of the Department of Biotechnology, India during the tenure of this study. A part of this work won the innocentive challenge competition designed for the development of urinary biomarkers for lupus nephritis.

\section{Author details}

'UND Life Sciences, 13800 Fairhill Road, \#321, Shaker Heights, OH 44120, USA. ${ }^{2}$ School of Biotechnology, Jawaharlal Nehru Technological University, Kakinada-533 003, India. ${ }^{3}$ Krishna Institute of Medical Sciences,

Secunderabad-500 003, India. ${ }^{4}$ Bio-Science Research Centre, GVP College of Engineering Campus, Madhurawada, Visakhapatnam-530 048, India.

\section{Conflict of interest statement}

The authors declare that they have no competing interests.

Received: 6 May 2011 Accepted: 15 May 2011 Published: 15 May 2011

\section{References}

1. Das UN: Clinical laboratory tools to diagnose inflammation. Advances in Clinical Chemistry 2006, 41:189-229.

2. Serhan CN: Lipoxins and aspirin-triggered 15-epi-lipoxins are the first lipid mediators of endogenous anti-inflammation and resolution. Prostaglandins Leukot Essent Fatty Acids 2005, 73:141-162.

3. Claria J, Serhan CN: Aspirin triggers previously undescribed bioactive eicosanoids by human endothelial cell-leukocyte interactions. Proc Natl Acad Sci USA 1995, 92:9475-9479.

4. Das UN: Essential fatty acids: Biochemistry, physiology, and pathology. Biotechnology J 2006, 1:420-439.

5. Serhan CN, Hong S, Gronert K, Colgan SP, Devchand PR, Mirick G, Moussignac R-L: Resolvins: a family of bioactive products of omega-3 fatty acid transformation circuits initiated by aspirin treatment that counter proinflammatory signals. J Exp Med 2002, 196:1025-1037.

6. Das UN: Essential fatty acids- a review. Current Pharmaceutical Biotechnol 2006, 7:467-482.

7. Chiang N, Arita M, Serhan CN: Anti-inflammatory circuitry: lipoxin, aspirintriggered lipoxins and their receptor ALX. Prostaglandins Leukot Essen Fatty Acids 2005, 73:163-177.

8. Levy BD, Clish CB, Schmidt B, Gronert K, Serhan CN: Lipid mediator class switching during acute inflammation signals in resolution. Nat Immunol 2001, 2:612-619.

9. Serhan CN, Maddox JF, Petasis NA, Akritopoulou-Zanze I, Papayianni A, Brady HR, Colgan SP, Madara JL: Design of lipoxin $\mathrm{A}_{4}$ stable analogs that block transmigration and adhesion of human neutrophils. Biochemistry 1995, 34:14609-14615.

10. Zordoky BN, El-Kadi AO: Effect of cytochrome P450 polymorphism on arachidonic acid metabolism and their impact on cardiovascular diseases. Pharmacol Ther 2010, 125:446-463.
11. Nithipatikom K, Gross GJ: Review article: epoxyeicosatrienoic acids: novel mediators of cardioprotection. J Cardiovasc Pharmacol Ther 2010, 15:112-119.

12. Campbell WB, Fleming I: Epoxyeicosatrienoic acids and endotheliumdependent responses. Pflugers Arch 2010, 459:881-895.

13. Arnold C, Konkel A, Fischer R, Schunck WH: Cytochrome P450-dependent metabolism of omega- 6 and omega-3 long-chain polyunsaturated fatty acids. Pharmacol Rep 2010, 62:536-547.

14. Kroetz DL, Zeldin DC: Cytochrome P450 pathways of arachidonic acid metabolism. Curr Opin Lipidol 2002, 13:273-283.

15. Gao L, Yin H, Milne GL, Porter NA, Morrow JD: Formation of F-ring isoprostane-like compounds (F3-isoprostanes) in vivo from eicosapentaenoic acid. J Biol Chem 2006, 281:14092-14099.

16. Serhan CN, Clish CB, Brannon J, Colgan SP, Chiang N, Gronert K: Novel functional sets of lipid-derived mediators with antiinflammatory actions generated from omega-3 fatty acids via cyclooxygenase 2-nonsteroidal antiinflammatory drugs and transcellular processing. J Exp Med 2000, 192:1197-1204.

17. Serhan CN, Clish CB, Brannon J, Colgan SP, Gronert K, Chiang N: Antimicroinflammatory lipid signals generated from dietary N-3 fatty acids via cyclooxygenase- 2 and transcellular processing: a novel mechanism for NSAID and N-3 PUFA therapeutic actions. J Physiol Pharmacol 2000, 51(4 Pt 1):643-654.

18. Marcheselli VL, Hong S, Lukiw WJ, Tian XH, Gronet K, Musto A, Hardy M, Gimenez JM, Chiang N, Serhan CN, Bazan NG: Novel docosanoids inhibit brain ischemia-reperfusion-mediated leukocyte infiltration and proinflammatory gene expression. J Biol Chem 2003, 278:43807-43817.

19. Hong S, Gronert K, Devchand PR, Moussignac RL, Serhan CN: Novel docosatrienes and 17S-resolvins generated from docosahexaenoic acid in murine brain, human blood, and glial cells. Autacoids in antiinflammation. J Biol Chem 2003, 278:14677-14687.

20. Romano M, Luciotti G, Gangemi S, Marinucci F, Prontera C, D'Urbano E, Davì G: Urinary excretion of lipoxin $\mathrm{A}(4)$ and related compounds: development of new extraction techniques for lipoxins. Lab Invest 2002, 82:1253-1254.

21. Gangemi S, Luciotti G, D'Urbano E, Mallamace A, Santoro D, Bellinghieri G, Davi G, Romano M: Physical exercise increases urinary excretion of lipoxin A4 and related compounds. J Appl Physiol 2003, 94:2237-2240

22. Gangemi S, Pescara L, D'Urbano E, Basile G, Nicita-Mauro V, Davì G, Romano M: Aging is characterized by a profound reduction in antiinflammatory lipoxin A4 levels. Exp Gerontol 2005, 40:612-614.

23. Wu SH, Liao PY, Yin PL, Zhang YM, Dong L: Inverse temporal changes of lipoxin A4 and leukotrienes in children with Henoch-Schönlein purpura. Prostaglandins Leukot Essent Fatty Acids 2009, 80:177-183.

24. Katoh T, Lakkis FG, Makita N, Badr KF: Co-regulated expression of glomerular 12/15-lipoxygenase and interleukin-4 mRNAs in rat nephrotoxic nephritis. Kidney Int 1994, 46:341-349.

25. Nassar GM, Badr KF: Role of leukotrienes and lipoxygenases in glomerular injury. Miner Electrolyte Metab 1995, 21:262-270.

26. Papayianni A, Serhan CN, Phillips ML, Rennke HG, Brady HR: Transcellular biosynthesis of lipoxin A4 during adhesion of platelets and neutrophils in experimental immune complex glomerulonephritis. Kidney Int 1995, 47:1295-1302.

27. O'Meara YM, Brady HR: Lipoxins, leukocyte recruitment and the resolution phase of acute glomerulonephritis. Kidney Int Suppl 1997, 58:S56-S61.

28. Wu SH, Liao PY, Yin PL, Zhang YM, Dong L: Elevated expressions of 15lipoxygenase and lipoxin A4 in children with acute poststreptococcal glomerulonephritis. Am J Pathol 2009, 174:115-122.

29. Lattin JE, Schroder K, Su Al, Walker JR, Zhang J, Wiltshire T, Saijo K, Glass CK, Hume DA, Kellie S, Sweet MJ: Expression analysis of G Protein-Coupled Receptors in mouse macrophages. Immunome Res 2008, 4:5.

30. Milligan G, Stoddart LA, Brown AJ: G protein-coupled receptors for free fatty acids. Cell Signal 2006, 18:1360-1365.

31. Oh DY, Talukdar S, Bae EJ, Imamura T, Morinaga H, Fan W, Li P, Lu WJ, Watkins SM, Olefsky JM: GPR120 is an omega-3 fatty acid receptor mediating potent anti-inflammatory and insulin-sensitizing effects. Cell 2010, 142:687-698.

32. Suresh $Y$, Das UN: Protective action of arachidonic acid against alloxaninduced cytotoxicity and diabetes mellitus. Prostaglandins Leukotrienes Essential Fatty Acids 2001, 64:37-52. 
33. Suresh $Y$, Das UN: Long-chain polyunsaturated fatty acids and chemically-induced diabetes mellitus: Effect of $\omega-6$ fatty acids. Nutrition 2003, 19:93-114.

34. Suresh $Y$, Das UN: Long-chain polyunsaturated fatty acids and chemically-induced diabetes mellitus: Effect of $\omega-3$ fatty acids. Nutrition 2003, 19:213-228.

35. Stene LC, Joner G, Norwegian Childhood Diabetes Study Group: Use of cod liver oil during the first year of life is associated with lower risk of childhood-onset type 1 diabetes: a large, population-based, case-control study. Am J Clin Nutr 2003, 78:1128-1134.

36. Stene $L C$, Ulriksen J, Magnus $P$, Joner G: Use of cod liver oil during pregnancy associated with lower risk of Type I diabetes in the offspring. Diabetologia 2000, 43:1093-1098.

37. Bellenger J, Bellenger S, Bataille A, Massey KA, Nicolaou A, Rialland M, Tessier C, Kang JX, Narce M: High Pancreatic $n-3$ fatty acids prevent STZinduced diabetes in fat-1 mice: inflammatory pathway inhibition. Diabetes.

38. White PJ, Arita M, Taguchi R, Kang JX, Marette A: Transgenic restoration of long-chain $\mathrm{n}-3$ fatty acids in insulin target tissues improves resolution capacity and alleviates obesity-linked inflammation and insulin resistance in high-fat-fed mice. Diabetes 2010, 59:3066-3073.

39. Das UN, Puskás LG: Transgenic fat-1 mouse as a model to study the pathophysiology of cardiovascular, neurological and psychiatric disorders. Lipids Health Dis 2009, 8:61

40. Bonventre JV: Phospholipase A2 and signal transduction. J Am Soc Nephrol 1992, 3:128-150.

41. Levy BD, Clish CB, Schmidt B, Gronert K, Serhan CN: Lipid mediator class switching during acute inflammation: signals in resolution. Nat Immunol 2001, 2:612-619.

42. Godson C, Mitchell S, Harvey K, Petasis NA, Hogg N, Brady HR: Cutting edge: lipoxins rapidly stimulate nonphlogistic phagocytosis of apoptotic neutrophils by monocyte-derived macrophages. J Immunol 2000, 164:1663-1667.

43. Gilroy DW, Colville-Nash PR, Willis D, Chivers J, Paul-Clark MJ, Willoughby DA: Inducible cyclooxygenase may have anti-inflammatory properties. Nat Med 1999, 5:698-701.

44. Gilroy DW, Newson J, Sawmynaden P, Willoughby DA, Croxtall JD: A novel role for phospholipase $A_{2}$ isoforms in the checkpoint control of acute inflammation. FASEB J 2004, 18:489-498

45. Cominelli F, Nast CC, Llerena R, Dinarello CA, Zipser RD: Interleukin 1 suppresses inflammation in rabbit colitis. Mediation by endogenous prostaglandins. J Clin Invest 1990, 85:582-586.

46. Schwab JH, Anderle SK, Brown RR, Dalldorf FG, Thompson RC: Pro- and anti- inflammatory roles of interleukin-1 in recurrence of bacterial cell wall-induced arthritis in rats. Infect Immun 1991, 59:4436-4442.

47. Serhan CN, Hong S, Gronert K, Colgan SP, Devchand PR, Mirick G, Moussignac R-L: Resolvins: A family of bioactive products of omega-3 fatty acid transformation circuits initiated by aspirin treatment that counter proinflammation signals. J Exp Med 2002, 196:1025-1037.

48. Croxtall JD, Choudhury Q, Tokumoto H, Flower RJ: Lipocortin-1 and the control of arachidonic acid release in cell signalling. Glucocorticoids inhibit G protein-dependent activation of CPLA2 activity. Biochem Pharmacol 1995, 50:465-474.

\section{doi:10.1186/1476-511X-10-76}

Cite this article as: Das: Lipoxins as biomarkers of lupus and other inflammatory conditions. Lipids in Health and Disease 2011 10:76.

\section{Submit your next manuscript to BioMed Central and take full advantage of:}

- Convenient online submission

- Thorough peer review

- No space constraints or color figure charges

- Immediate publication on acceptance

- Inclusion in PubMed, CAS, Scopus and Google Scholar

- Research which is freely available for redistribution

Submit your manuscript at www.biomedcentral.com/submit
C Biomed Central 